\title{
The small species of Belostoma (Heteroptera: Belostomatidae): Revision of plebejum group
}

\author{
Ana Lía Estévez ${ }^{1} \&$ John T. Polhemus ${ }^{2}$ \\ 1 División Entomología. Museo de La Plata. Paseo del Bosque s/nº 1900 La Plata. Argentina; \\ estevezanalia@hotmail.com \\ 2 University of Colorado Museum, 3115 S. York St. Englewood, Colorado, USA 80110.
}

Received 10-X-2005. Corrected 10-III-2006. Accepted 23-V-2006.

\begin{abstract}
Nieser gathered the water bug species included by Lauck in the groups Belostoma pygmeum and Belostoma plebejum in a single group, Belostoma plebejum. We follow this criterion and use the terminology used by Estévez and Polhemus for species descriptions. The Belostoma plebejum group includes seven small species with the ventral diverticulum of male genitalia recurved ventrad: Belostoma lariversi De Carlo, 1969; B. micantulum (Stål, 1858); B. plebejum (Stål, 1858); B. pygmeum (Dufour, 1863); B. minusculum (Uhler, 1884). B. parvum sp. nov. from Venezuela, Colombia, Guyana, Suriname and Brazil, and B. nicaeum sp. nov. from Manaus, Brazil. The two new species are described and illustrated, and new synonymies are proposed. Rev. Biol. Trop. 55 (1): 147-155. Epub 2007 March. 31.
\end{abstract}

Key words: Neotropical, taxonomy, Belostoma, small species, plebejum group.

Lauck (1962) included the small water bug species that have a ventroapical protuberance in the ventral diverticulum of male genitalia, in the Belostoma plebejum group, and the species with the ventral diverticulum with thickened lateral margins in the Belostoma pygmeum group. This author distinguished the groups within a key, but did not fully describe the species. Nieser (1975) considered that the margins of male genitalia's ventral diverticulum thickened represent a specialization of some species in the Belostoma pygmeum group and he included the species from both groups proposed by Lauck (1962) in a single group, Belostoma plebejum. Herein Nieser's criteria is followed, we consider that the main characteristic of the species in this group is having the ventral diverticulum of male genitalia recurved ventrad. We make the revision of seven species recognized for the B. plebejum group, from which two are herein described as new, and new synonymies are proposed.

\section{MATERIAL AND METHODS}

The examined material was borrowed from the following institutions: California Academy of Sciences, San Francisco, USA (CAS); John T. Polhemus Collection, Denver, USA (JTPC); Los Angeles County Museum, Los Angeles, USA (LACM); Francis Huntington Snow Entomological Collections, Kansas University, Lawrence, USA (KU); National Museum of Natural History, Washington D.C., USA (USNM); University of Arizona, Tucson, USA (UA); Museo Argentino de Ciencias Naturales "B. Rivadavia", Buenos Aires, Argentina (MACN); Museo de La Plata, La Plata, Argentina. (MLP).

Taxonomic characters used in the descriptions are the same mentioned by Estévez and Polhemus (2001).

\section{Belostoma plebejum group}

Size: length, 9.5-16.2 mm; width, 5.0-7.0 $\mathrm{mm}$. Shape: ovate. Color: light to dark brown. 
Head: suturae anteclypeus-maxillary plate as long as suturae anteclypeus-lorum; anteoculus shorter than interoculus; eyes globose, as wide as long; segment I of the beak shorter or as long as segment II. Thorax: prosternal keel rounded or with anterior margin straight.
Abdomen: pilosity covering less than half of ventral laterotergites, strongly constricted between spiracles, slightly developed on the penultimate visible segment.

Male genitalia: ventral diverticulum recurved ventrad.

\section{Key to the species of $B$. plebejum group}

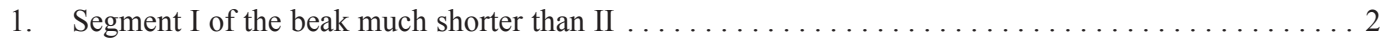

1 '. Segment I of the beak as long as II or slightly shorter than II $\ldots \ldots \ldots \ldots \ldots \ldots \ldots \ldots \ldots$

2. Prosternal keel rounded, not projected anterad. Length of the body much more than $11 \mathrm{~mm} \ldots \ldots \ldots 3$

2'. Prosternal keel acute, slightly projected anterad. Length of the body ranging from 9.50 to $11.10 \mathrm{~mm}$ Belostoma parvum sp.nov.

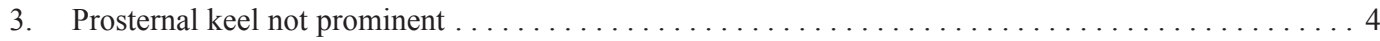

3'. Prosternal keel prominent . . . . . . . . . . . . . . . . . . . . . . . . . . . . . . . . . 5

4. Length of the body twice or more the width of the body; abdominal pilosity (Fig. 30); ventral diverticu-

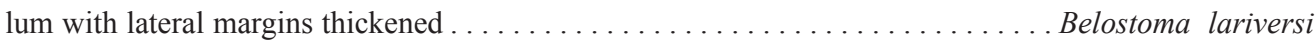

4'. Length of the body less than twice the width of the body; abdominal pilosity (Fig. 34); ventral diverticulum with lateral margins not thickened . . . . . . . . . . . . . . . . . Belostoma micantulum

5. Length of the body ranging from 11.40 to $13.70 \mathrm{~mm}$; abdominal pilosity (Fig. 38); ventral diverticulum with lateral margins thickened........................... Belostoma nicaeum sp. nov.

5'. Length of the body more than $13 \mathrm{~mm}$; abdominal pilosity (Fig. 42); ventral diverticulum with lateral margins not thickened Belostoma plebejum

6. Prosternal keel not prominent; ventral diverticulum with lateral margins thickened

Belostoma pygmeum

6'. Prosternal keel prominent; ventral diverticulum with lateral margins not thickened .Belostoma minusculum

\section{Belostoma parvum sp. nov.}

(Fig. 1-3, 22-25)

Size: length, or $9.50 \mathrm{~mm}-10.70 \mathrm{~mm}$, o $9.60 \mathrm{~mm}-11.10 \mathrm{~mm}$; width, o $^{7} 4.60 \mathrm{~mm}-5.50$ $\mathrm{mm}$, o $4.80 \mathrm{~mm}-5.90 \mathrm{~mm}$. Color: yellowish brown. Shape: elliptical (Fig. 1).

Head: suture anteclypeus-maxilary plate as long as suture anteclypeus-lorum; anteoculus shorter than interoculus; eyes globose,as wide as long (Fig. 2); segment I of the beak shorter than II (Fig. 3). Thorax: prosternal keel rounded, not prominent (Fig. 3). Abdomen: pilosity developed on the penultimate segment, reaching the external margin (Fig. 22).

Male genitalia: arms of phalobase short, covering the external margin of ventral diverticulum; ventral diverticulum recurved ventrad (Fig. 23-25).

Remarks. This is the smallest species in the genus.

Distribution. Surinam, Guyana, Venezuela, Colombia and Brazil.

Types. Holotype $\sigma^{7}$, allotype $\bigcirc$ and 57 paratypes (36 $\sigma^{7}$ and $21 \%$ ) from Guyana, bearing the label "British Guiana, Suppuruni Creek, Aug. 20,1937, S. Harris" (JTPC).

Etymology: the name refers to the small size of these insects.

Specimens examined. SURINAM, Moengo, 1927, ․ (JTPC). GUYANA, Supuruni Creek, ơ, o. 1937, Harris coll. (JTPC). VENEZUELA, 
Portuguesa: Guanare, ơ', ㅇ. IX-1957, Menke coll. (CAS). COLOMBIA, Amazonas: Leticia, ○', 1974. Sedlacek coll. (JTPC). BRAZIL. Santarem, O' (LACM). O' Idem (JTPC).

\section{Belostoma lariversi De Carlo}

(Fig. 4-6, 26-29)

Belostoma lariversi De Carlo, 1960: 50-52, Fig. 7-99, 16. Lanzer- de -Souza, 1980: 58.

Size: length, ơ ᄋ $15.00 \mathrm{~mm}-17.00 \mathrm{~mm}$; width, ơ o $7.00 \mathrm{~mm}-7.50 \mathrm{~mm}$. Color: yellowish brown. Shape: ovoid (Fig. 4).

Head: suture anteclypeus-maxilary plate as long as suture anteclypeus-lorum; anteoculus shorter than interoculus; eyes globose, as wide as long (Fig. 5); segment I of the beak shorter than II (Fig. 6). Thorax: prosternal keel rounded, not prominent (Fig. 6). Abdomen: pilosity slightly developed on the penultimate segment (Fig. 26).

Male genitalia: arms of phalobase short, not covering the external margin of ventral diverticulum; ventral diverticulum recurved ventrad, margins thickened (Fig. 27-29).

Remarks. This species is similar in shape to $B$. micantulum but male genitalia has lateral margins of ventral diverticulum thickened.

Distribution. From the Type locality Pan de Azúcar, Depts.Pasco and Huanuco, Cucharas. (Peru).

Types. Holotype $\sigma^{7}$, allotype $\bigcirc$ and 4 paratypes $\left(\sigma^{\top}\right.$ and 3 \%) deposited in USNM in C.J. Drake Collection, bearing label "Peru, Cucharas, VIII-1954. (Cucharas, Fuallago)" and "Peru, Cucharas, leg. P.L.W., VI-1954 Cucharas, Pacaya". We have examined two paratypes $\left(\sigma^{\top}\right.$,, ) from the type locality deposited in MACN.

Specimens examined. PERU, Pasco: Pan de Azúcar, VIII-1961, Truxal coll. 35 ช , 28 ․ (LACM). Huanuco: Tingo Maria, $17 \sigma^{7}$ , 10 \&, VI.1982, Challet coll. (LACM). Rio Marañón, Ơ IV.1924, Basler coll. (JTPC).

\section{Belostoma micantulum (Stål)}

(Fig. 7-9, 30-33)

Zaitha micantula Stål, 1860: 84. Mayr, 1871: 420-421, (in part); Walker, 1873: 120, (in part); Berg, 1879: 190, (in part); Champion, 1901:366, (in part). Zaitha zelotypus White, 1879: 270. Belostoma micantulum: Kirkaldy \& Torre Bueno, 1909: 191-192, (in part, = zelotypus); De Carlo, 1930: 119-120, (in part); 1938: 228, (in part); Nieser, 1975: 115; Schnack , 1976: 38; Lanzer-de- Souza, 1980: 59-60.

Belostoma husseyi De Carlo, 1960: 48, Fig. 2-6; Lanzer-de-Souza, 1980: 58. SYN. NOV. Belostoma apache: De Carlo, 1960: 53 (misidentification).

Size: length, or $10.80 \mathrm{~mm}-13.70 \mathrm{~mm}$, o $10.70 \mathrm{~mm}-15.10 \mathrm{~mm}$; width, or $5.40 \mathrm{~mm}-6.80$ $\mathrm{mm}$, ᄋ $5.20 \mathrm{~mm}-7.10 \mathrm{~mm}$. Color: light to dark brown. Shape: elliptic (Fig. 7).

Head: suture anteclypeus-maxilary plate as long as suture anteclypeus-lorum; anteoculus shorter than interoculus; eyes globose, as wide as long (Fig. 8); segment I of the beak shorter than II (Fig. 9). Thorax: prosternal keel rounded, not prominent (Fig. 9). Abdomen: pilosity slightly developed on the penultimate segment, extending to the external margin of the laterotergite (Fig. 30).

Male genitalia: arms of phalobase covering the external margin of ventral diverticulum; ventral diverticulum with a deep depression in the mid dorsal line (Fig. 31-33).

Remarks. The accurate identification of this species is only possible through the male genitalia, because of its deep depression in the mid dorsal line of ventral diverticulum.

Types and synonymy. We have examined the holotype of $B$. husseyi and we have not found differences between this species and B. micantulum. External characteristics vary among individuals from the same population, so we propose the synonymy of $B$. husseyi with B. micantulum. 


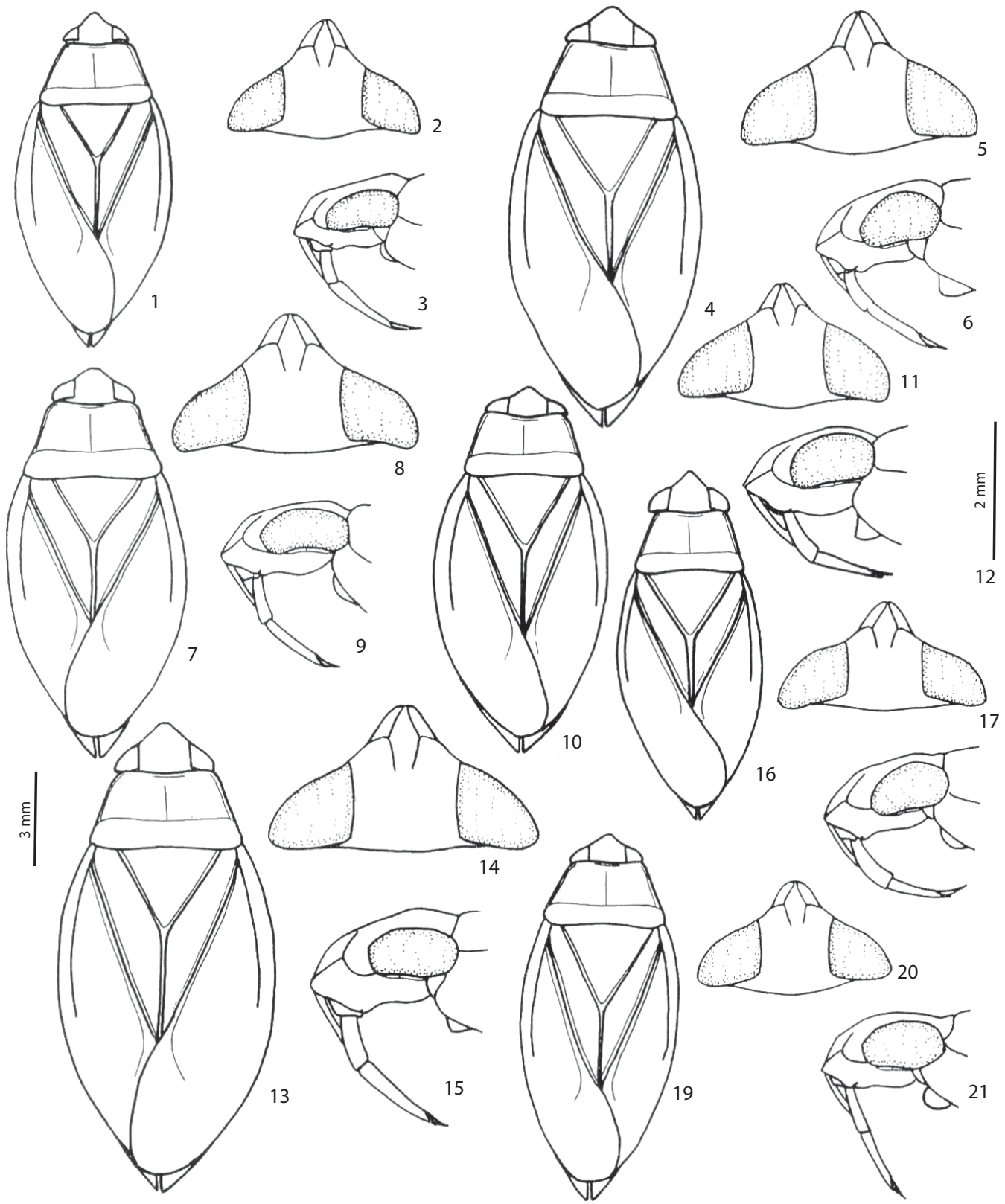

Fig. 1-21. Body form, head dorsal and lateral views: 1-3, Belostoma parvum sp. nov.; 4-6, B. lariversi De Carlo; 7-9, B. micantulum (Stål); 10-12, B. nicaeum sp. nov.; 13-15, B. plebejum (Stål); 16-18, B. pygmeum (Dufour); 19-21, B. minusculum (Uhler).

Distribution. Widely distributed in South America: Guyana, Venezuela, Colombia, Brazil, Paraguay, Bolivia and Argentina.

Type. A male which is deposited in Stockholm Museum and bears the label: "Rio
Jan." is herein designated as lectotype. Our lectotype label has been added.

Specimens examined. VENEZUELA, Barinas, Pte. Gen. Paez, Río Bocono, q, IX.1965, B. Malkin coll. (CAS); San Esteban, 
6 ơ, 7 ९, XI.1939, P.Anduze coll. (JTPC); Monagas, 60 km SE Maturín, 44 ○', 27 ๆ, 17.VI5.VII. 1958, Menke coll. (JTPC); Caracas, or, ๆ (USNM); COLOMBIA, Cuatro Bocas, q, I.1959, (USNM); Amazonas, Leticia, q, VI.1965, P. Craig-J.Robb coll. (CAS); BRAZIL, Rio Grande do Sul: Itahim, 4 o', I.1947, Wygodzinsky (USNM). Goias: Sta. Isabel, Ilha do Bananal, Rio Araguaia, o,VIII.1957, Malkin coll. (CAS); side Rio Araguaia, $3 \mathrm{~km}$ S Barra do Tapirapé, B.Malkin coll., $q$, (CAS); Mato Grosso: Barra do Tapirapé, \&,XII.1962, B. Malkin (CAS); Porto Velho, Rio Tapirapé, ơ, 11.VII.1962, R.Pinheiro coll (CAS); Jacaré PN Xingu, 16 ○', XI.1965, Alvarenga coll. 17 क, (LACM); 12 ○', 8 ๆ, XI.1961, Alvarenga coll. (LACM); 124 km S. Peixe, Go., $3 \sigma^{7}, 5$ \%, VI.1956, Truxal coll. (LACM); 48 km S. Peixe, Go., 10', VI.1956, Truxal coll. (LACM); 3 km S. Peixe, Go., 10', VI.1956, Truxal coll (LACM); 34 km S. Amaro Leite, Go.,1 o', V/1958, Truxal coll. (LACM); 20 km N. São João da Aliança, GO., ơ, IV.1956, Truxal coll. (LACM); PARAGUAY, Sapucay, XII.1902, Heldemann coll. 3 o, (CU). Primavera, or, , I.1956, A. Menke coll.. (LACM); Emboscada, ○’, ᄋ, II.1954 , A. Menke coll. (LACM); Alto., o', O, IX.1953, A. Menke coll. (LACM); Colonia Ferheim, Chaco, 19 o', 10 \&, XI.1956, A. Menke coll. (LACM); Trinidad, $\sigma^{7}$, XII.1920, Jorgensen coll. (JTPC); Villarica, q, IV.22.1950, Schade coll. (JTPC); BOLIVIA, Chapare, Río Chipiriri, 2 o , 4 ๑, XI.1953, W. Foerster coll. (JTPC); Espíritu, Río Yacuma, O', VII.1950, W. Foerster coll. (JTPC); Santa cruz, Steinbach coll., 3 o', (JTPC); ARGENTINA, Salta: Pocitos, , II.1952, A.Menke coll. (LACM); Orán, Aguas Blancas, O', II.1960, Vollenweider coll. (LACM); Urundel, $\sigma^{7}$, X.1968, L.Peña coll. (JTPC); Tucumán: 4 ○', 6 9, (MLP); Santiago del Estero: El Pinto, 5 o', 1 Q, XI.1956, A. Menke coll. (LACM); Formosa: 6 o $^{7}, 5$ o, XII.1954, A. Menke coll. (LACM); Gran Guardia, 103 o', 89 , XI.1952, A. Menke coll. (LACM); Gran Guardia, 12 ơ, 8 \%, XI.1952, Foerster coll. (USNM); Guayacolec, 554 ○’,49 , XII.1956, A. Menke coll. (LACM); Misiones: Apóstoles,
2 ơ, 6ᄋ, II.1979 (UA); Apóstoles, 1 ○', IX.1979 (UA); Panambí, 12 ơ, 5 ㅇ, XII.1957, A.Menke coll. (LACM); Chaco: Resistencia, 3 ○', 6 \%, XI.1948, Martínez coll. (JTPC); Resistencia, 1 o', 1 \%, 2.XII.1939, Birabén \& Bezzi coll. (MLP); Pto. Barranqueras, 3 ○, 4 १, 2.XII.1939, Birabén \& Bezzi coll. (MLP); Corrientes: Paso de la Patria, 74 o', 79 o , XII.1954, Menke coll. (LACM); Santa Fe: Reconquista, o', 2 ᄋ, 22.XII.1939, M. Parko leg. (MLP); Rosario, 9 o', 17 , I.1925, A. Ligreski coll. (USNM); Esperanza, Reimoser coll., $1 \sigma^{7}$, (USNM); Santo Tomé, Río Salado, 6 o', 8 \%, III.1971, Flint coll. (USNM); Villa Guillermina, O', II.1959 (JTPC); Entre Ríos: La Paz, 12 ơ', 10 ९,VII.1949, Foerster coll.(JTPC); Paraná, O7, 6 ९, III.1948, A. Menke coll. (LACM); Pronunciamiento, $23 \sigma^{7}$, 27 o, I/1964, (LACM); Puerto Marquez, 8 ơ, 9 ,VIII.1951, A. Menke coll. (LACM).

\section{Belostoma nicaem sp. nov.}

(Fig. 10-12, 34-37)

Size: length, or $11.80 \mathrm{~mm}-13.10 \mathrm{~mm}$, o $11.40 \mathrm{~mm}-13.70 \mathrm{~mm}$; width, or $5.50 \mathrm{~mm}-6.00$ $\mathrm{mm}$, ㅇ $5.80 \mathrm{~mm}-6.20 \mathrm{~mm}$. Color: brown mottled with yellow. Shape: oval (Fig. 10)

Head: suture anteclypeus-maxilary plate as long as suture anteclypeus-lorum; anteoculus shorter than interoculus; eyes globose, as wide as long (Fig. 11); segment I of the beak shorter than II (Fig. 12). Thorax: prosternal keel acute, projected anterad (Fig. 12).Abdomen: pilosity slightly developed on the penultimate segment (Fig. 34).

Male genitalia: arms of phalobase divergent, lateral margins of ventral diverticulum thickened (Fig. 35-37).

Remarks. This species is easily distinguished from the other species included in this group by the acute prosternal keel and the divergent arms of the phalobase.

Distribution. Only recorded from type locality.

Type. Holotype $\sigma^{\top}$, alotype $O$ and 15 paratypes ( $8 \sigma^{7}$ and 79 ), bearing the label "Brazil S.A., Aug. 1935, A. M. Olalla", "Rio 
Negro, Manaus Region” (Manaus, Amazonas). Deposited in UK. Three paratypes (2 $O^{\top}$ and $\%$ ) in JTPC.

Other specimens examined. BRAZIL, Rio Negro, Manaus Region, 11 ơ', 11 \&,Aug. 1935, A. M. Olalla coll. (JTPC).

Etimology: the name refers to these specimens as to being nice.

\section{Belostoma plebejum (Stål)}

(Fig. 13-15, 38-41)

Zaitha plebeja Stål, 1858: 83-84. Mayr, 1863: 353-354; 1871: 418-420, (in part); Walker, 1873: 120 (in part); Montandon, 1895: 10. Zaitha micantula: Dufour, 1863: 391 (probably). Belostoma plebejum: Kirkaldy \& Torre Bueno, 1909:192, (in part). Belostoma plebejum: De Carlo, 1930: 118-119, (in part); 1938: 227-228, (in part); Schnack, 1976:40,(part); Lanzer-de-Souza, 1980: 61-62 (part).

Size: length, or $14.20 \mathrm{~mm}-15.90 \mathrm{~mm}$, of $13.70 \mathrm{~mm}-16.20 \mathrm{~mm}$; width, or $^{7} 7.30 \mathrm{~mm}-7.60$ $\mathrm{mm}$, o $6.60 \mathrm{~mm}-7.60 \mathrm{~mm}$. Color: yellowish brown to dark brown. Shape: elliptic (Fig. 13).

Head: suture anteclypeus-maxilary plate as long as suture anteclypeus-lorum;anteoculus shorter than interoculus; eyes globose,as wide as long (Fig. 14); segment I of the beak shorter than II (Fig. 15). Thorax: prosternal keel prominent, with the anterior margin straight, projected anterad (Fig. 15). Abdomen: pilosity slightly developed on the penultimate segment (Fig. 38).

Male genitalia: arms of phalobase not covering the external margin of ventral diverticulum, ventral diverticulum with a deep depression on dorsal mid line, strongly recurved ventrad (Fig. 39-41).

Remarks. B. plebejum is similar in shape to B. micantulum, but the first mentioned species is more robust.

Distribution. This species are recorded from Peru, Bolivia, southern Brazil, Argentina and Uruguay.

Type. According with Lauck (1955) this species was described on a specimen labeled "Rio Jan., Typus, Stål", identified as
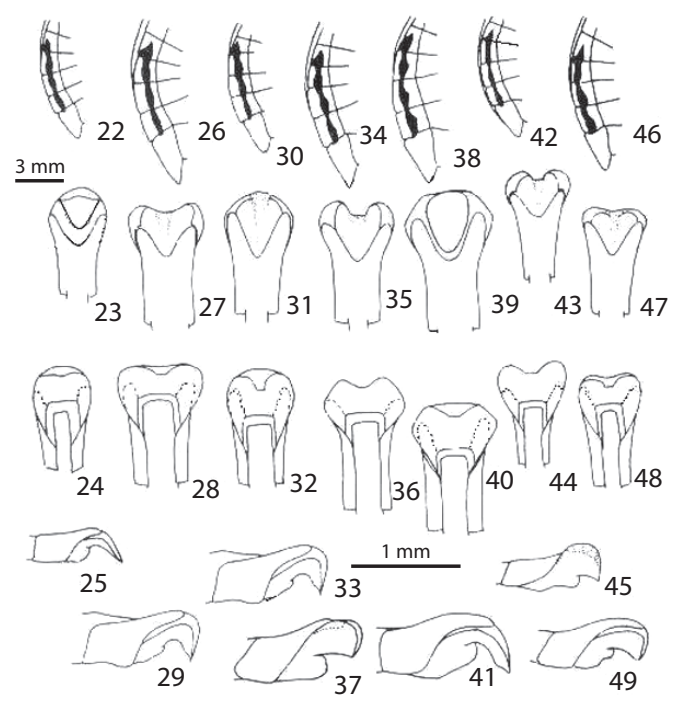

Fig. 23-49. Abdominal pilosity and male genitalia dorsal, ventral and lateral views: $22-25, B$. parvum; 26-29, $B$. lariversi; 30-33, B. micantulum; 34-37, B. nicaeum; 38-41, B. plebejum; 42-45, B. pygmeum; 46-49, B. minusculum.

B. plebejum. deposited in Stockholm Museum, which we examined..

Specimens examined. PERU, Río Marañón, O`, o, IX.1924, Bassler coll. (JTPC); Quincemil, Peña coll., 2 ○, $q$ (JTPC). BRAZIL, Rio de Janeiro: D.F., Wygodzinsky coll., H, (JTPC); Lag. de Juturnaiba, Araruana, I.1977, SantosMatos coll., O', 4 ๆ (JTPC); 20 km São João da Aliança, Truxal coll. 2 ○', IV.1956 (LACM); São Carlos, Aguas Mineraes, 3 o', 5 @ V/1957 (LACM); Rio S. Paulo km 47, 2 ○', o,I.1944, Wygodzinskky (USNM); Sta. Catarina: Nova Teutonia, 4 o,VIII.1953, Plaumann (USNM); o', VIII.1932 to I.1933, Jhon Thaden, Holst coll. (JTPC); 27 11B 5223 L, 3 O', o, Plaumann coll. (LACM). PARAGUAY; Villarica, 8 O', 7 \%, IX.19923, F.Scade coll. (JTPC); 1947, F.Schade coll. @ (JTPC); Alvobena, Srojoguasi,, , XII.1926, F.Schade coll. (JTPC); ARGENTINA, Tucumán: O', \%, 9.III.1939 (MLP). Río Lules, O', X.1932 (MLP): Santa Fe: Rosario. P. Denier col., O', ᄋ (MLP); Entre Ríos: 2 o', 2 क, XII.1940 (MLP); Buenos Aires:Bosq coll. $\sigma^{\top}$,, , (MLP). URUGUAY, Tacuarembó; Pta. Arroyo laureles, O', I.1954, F.N.C. (LACM). 
Belostoma pygmeum Dufour

(Fig. 16-18, 42-45)

Zaitha pygmea Dufour, 1863: 228; Mayr, 1871: 421-422; Walker, 1873: 180. Belostoma pygmeum: Kirkaldy \& Torre Bueno, 1909: 192; Nieser, 1975: 118; Lanzer-de-Souza,1980: 62. Belostoma micantulum: De Carlo, 1930: 119120 (in part); De Carlo, 1938: 228 (in part). Belostoma menkei De Carlo, 1960: 52, Fig. 1013, 15; Lanzer-de-Souza, 1980:59. SYN. NOV.

Size: length, or $10.50 \mathrm{~mm}-12.00 \mathrm{~mm}$, ㅇ $10.50 \mathrm{~mm}-13.00 \mathrm{~mm}$; width, or $5.00 \mathrm{~mm}-5.50$ $\mathrm{mm}$, o $4.50 \mathrm{~mm}-6.00 \mathrm{~mm}$. Color: brown. Shape: elliptic, elongated (Fig. 16).

Head: suture anteclypeus-maxilary plate as long as suture anteclypeus-lorum; maxilary plate slightly compressed; anteoculus shorter than interoculus; eyes globose, as wide as long (Fig. 17); segment I of the beak as long as II (Fig. 18). Thorax: prosternal keel rounded, not prominent (Fig. 18). Abdomen: pilosity slightly developed on the penultimate segment (Fig. 42).

Male genitalia: arms of phalobase short, not covering the external margin of ventral diverticulum, lateral margins of ventral diverticulum recurved dorsad (Fig. 43-45).

Remarks. B. pygmeum is easily distinguished from the other members of this group for having the lateral margins of ventral diverticulum recurved dorsad.

Types and synonymy: we have studied the types and additional specimens identified as B. menkei, which is synonymized with $B$. pygmeum, because the differences found among the examined material can be considered as individual variations.

Distribution. Río Paraguay basin in Peru, Paraguay, Bolivia and Brazil.

Type. We have examined the holotype $\bigcirc$ of B. pygmeum labeled "Pondich. Coll. Signoret". It is deposited in VM and the holotype of $B$. menkei deposited in USNM.

Specimens examined. PERU, Vic. San Pedro, O', V.1935, Woytkowski coll. (JTPC). Satipo, or, $ๆ$ (USNM); PARAGUAY, Centurión, Reimoser coll., 4 o', 4 ᄋ (USNM); San Luis,
Reimoser coll. Oð, 5 ๆ (USNM); Santa Sofía, Reimoser coll., o (USNM); Molinasque, q, X./1925, F.Schade coll. (JTPC); Horqueta, 2 o', I.1927, Schulze \& Drake coll. (LACM); $45 \mathrm{mi}$ E, 10 o', 14 \&, VII.1935, Schulze coll. (USNM); BOLIVIA, Santa Cruz: Nuflo de Chavez, $180^{7}$, 29 \&, XI.1963 (LACM); Del Sara, Steinbach, ơ (LACM); Reyes, Beni River, 2 \&, XI.1956, L.Peña coll. (JTPC).

\section{Belostoma minusculum (Uhler)}

(Fig. 19-21, 46-49)

Zaitha minuscula Uhler, 1884: 258 (in part, only specimens from Nicaragua); Champion, 1901: 366 (in part). Belostoma micantulum: Torre Bueno, 1906: 55; Kirkaldy \& Torre Bueno, 1909: 191-192 (in part); De Carlo, 1938: 228 (in part). Belostoma drake De Carlo, 1950: 530-531; 1960: 56, Fig. 20, 27-32. Lanzer-de-Souza, 1980: 54. SYN. NOV.

Size: length, Or $12.30 \mathrm{~mm}-14.00 \mathrm{~mm}$, o $11.50 \mathrm{~mm}-14.00 \mathrm{~mm}$; width, or $5.70 \mathrm{~mm}-6.70$ $\mathrm{mm}$, $95.60 \mathrm{~mm}-7.20 \mathrm{~mm}$. Color: dark brown. Shape: ovoid (Fig. 19).

Head: suture anteclypeus-maxilary plate as long as suture anteclypeus-lorum; anteoculus shorter than interoculus; eyes globose, as wide as long (Fig. 20); segment I of the beak slightly shorter than II (Fig. 21). Thorax: prosternal keel rounded, prominent (Fig. 21). Abdomen: pilosity slightly developed on the penultimate segment (Fig. 46).

Male genitalia: arms of phalobase not covering the external margins of ventral diverticulum, ventral diverticulum with a deep depression on middle dorsal line, strongly recurved ventrad (Fig. 47-49).

Remarks. Belostoma. minusculum is easily distinguished by having arms of phallobase not covering the lateral margins of ventral diverticulum, ventral diverticulum with a deep depression on the mid dorsal line.

Distribution. This species is recorded from Trinidad, Costa Rica, Nicaragua, Honduras, Panamá and Venezuela.

Types and synonymy. A male specimen labeled "Nicaragua", deposited in USNM is 
herein designated as lectotype. We have examined $B$. drakei, deposited in USNM which is synonymized with $B$. minusculum, because the only difference we found, among the examined specimens, is color; we consider that it is not a good character for defining Belostoma species.

Specimens examined. COSTA RICA, Guan. Prov.m, Finca Jiménez, Ag. Sta. Nr. Finca Tobago, ơ', 2 ๆ,II.III.1968, M.C.Diarmid coll. (LACM); Coto, Finca 44, 3 ○, \&, VIII.1957, A.Menke coll. (LACM); San Isidro de Gral., O', XI.1962, C.Hogue coll. (LACM); Farm Hamburg, aam. Reventazón, O’, III.1934 (LACM); 16 mi. S La Cruz, 9 o, 7 , VII.1965, Spangler coll. (USNM); Puntarenas, $2 \sigma^{\top}$, , VII.1965, Spangler coll. (USNM); Turrialba, 2 O$^{7}, 6$ \&, VII.1965, Spangler coll. (USNM). HONDURAS, $10 \mathrm{mi}$ W. Choluteca, 2 ơ $^{\top}$, , VII.1965, Spangler coll. (USNM). NICARAGUA, Bonanza Pond, O7, X.1955, B.Malkin coll. (LACM); Somoto, 2 o',, , VII.1965, Spangler coll. (USNM); La Trinidad, q, VII.1965, Spangler coll. (USNM); $22 \mathrm{mi}$ S Rivas, 2 ○, 5 o, VII.1965, Spangler coll. (USNM); 13 mi N San Benito, 2 o', , , VII.1965, Spangler coll. (USNM). PANAMA, Barro Colorado Isl., 3 o', 2 \%, V.1964, Duckworth coll. (USNM); Barro Colorado Isl. C.Z., 2 q, IV.1941 (USNM); Madden Dam, C.Z., 3 ○, 2 ○, V.1937, Bliss (USNM); Pte. Obaldía, ○', XI.1952, Blanton (USNM); La Chorrera, ơ, V.1912, Burk (USNM); Mojinga, ९, I.1953, Blanton (USNM); Coraza, , V.1912, Burk (USNM). TRINIDAD, Barataria, Cress Bed, o', IV.1949, White coll. (LACM). VENEZUELA, Miranda, Barlovento, Higuerote, 2 o', I.1962, Bordon coll. (LACM); Guarico, El Sombrero, ○', I.1961, Bordón coll. (LACM); Barinitas, o', q (USNM).

\section{RESUMEN}

Nieser reunió las especies de chinches acuáticas incluidas por Lauck en los grupos Belostoma plebejum y Belostoma pygmeum en un solo grupo, Belostoma plebejum, criterio que se sigue en este trabajo. El grupo incluye siete especies pequeñas que presentan el divertículo ventral de los genitales masculinos recurvado ventrad: Belostoma lariversi De Carlo, 1969; B. micantulum (Stål, 1858); B. plebejum (Stål, 1858); B. pygmeum (Dufour, 1863); B. minusculum (Uhler, 1884), las cuales se redescriben e ilustran y B. parvum registrada de Venezuela, Colombia, Guyana, Suriname y Brazil y B. nicaeum registrada de Manaus, Brasil. Las dos especies nuevas que se describen e ilustran.

Palabras claves: Neotropical, chinche acuática, taxonomía, Belostoma, especies pequeñas, grupo plebejum.

\section{REFERENCES}

Berg, G. 1879. Hemiptera argentina enumeravitt speciesque novas. An. Soc. cient. Argent. Buenos Aires. 316 p.

Champion, G.C. 1901. Insecta, Rhynchota. Hemiptera Heteroptera. In: Godman \& Salvin (eds.). Biologia Centrali Americana, v. 2. Taylor and Francis, London. $416 \mathrm{p}$.

De Carlo, J.A. 1930. Familia Belostomatidae, géneros y especies para la Argentina. Rev. Soc. Ent. Arg. 13: 101-124.

De Carlo, J.A. 1938. Los Belostómidos Americanos Hemiptera. An. Mus. Arg. Cienc. Nat. 39: 81-252.

De Carlo, J.A. 1950. Descripción de especies nuevas de Ranatridae y Belostomatidae y algunas aclaraciones referentes a otras poco conocidas. (Hemiptera). Rev. Bras. Biol. 10: 521-532.

De Carlo, J.A. 1960. Especies nuevas del género Belostoma y consideraciones sobre otras poco conocidas (Hemiptera: Belostomatidae). Rev. Soc. Ent. Arg. 22: 47-59.

Dufour, L. 1863. Essai Monographique sur les Belostomatides. An. Soc. Ent. Fr. 3: 373-400

Estévez, A.L. \& J.T. Polhemus. 2001. The small species of Belostoma (Heteroptera: Belostomatidae). Key to species groups and a revision of the denticolle group. Iheringia, Sér. Zool. 91: 151-158.

Kirkaldy, G. W. \& J.R. de la Torre Bueno. 1909. A Catalogue of American Aquatic and Semiaquatic Hemiptera. Proc. Ent. Soc. Wash. 10:173-215.

Lanzer-de-Souza, M.E. 1980. Inventário da distribução geográfica da familia Belostomatidae Leach, 1815 (Hemiptera-Heteroptera) na Região Neotropical. Iheringia, Sér. Zool. 55: 43-86.

Lauck, D.R. 1962. A monograph of the genus Belostoma (Hemiptera). Part I. Introduction and B.dentatum and subspinosum groups. Bull. Chicago Acad. Sci. 11: $34-81$. 
Mayr, G. 1863. Hemipterologische Studien. Die Belostomiden. Verh. Zool.-bot. Ges. Wien. 13: 339364.

Montandon, A.L. 1871. Die Belostomiden. Verh. Zool.-bot. Ges. Wien. 21: 399-440.

Montandon, A. L. 1895. Hémiptères Hétéroptères. Première liste et descriptions d' espèces nouvelles. Viaggio del Dott. A.Borelli nella Republica Argentina en el Paraguay. Bull. Mus.. Zool. Anat. Comp. R. Univ. Torino 10:1-10.

Nieser, N. 1975. The water bugs (Heteroptera-Nepomorpha) of the Guyana Region. Stud. Fauna Suriname Univ. Utrecht 16: 1-310.

Schnack, J.A. 1976. Insecta-Hemiptera-Belostomatidae, p. 1-66. In: Fundación para la educación, la ciencia y la cultura. Fauna de Agua Dulce de la República Argentina. Buenos Aires. 35 Fasc. 1.
Stål, C. 1860 (1858). Bidrag till Rio de Janeiro traktens Hemipter fauna. K. svenska VetensksAkad. Handl. N. F. 2: 1-84.

Stål, C. 1862. Bidrag till Rio de Janeiro traktens Hemipter fauna. K. svenska VetensksAkad. Handl. N. F. 6: 1-75.

Torre Bueno, J. R. de la. 1906. Life histories of North American water bugs. I. Life history of Belostoma fluminea Say. Can. Ent. 38: 189-197.

Uhler, P. R. 1884. Order IV. Hemiptera. Stand. Nat. Hist. 2: 204-296.

Walker, F. 1873. Catalogue of the specimens of Hemiptera Heteroptera in the collection of the British Museum. British Museum, London. Part 8, 120 p.

White, F.B. 1879. List of the Hemiptera collected in the Amazons by Prof. I.W.H. Trail, M.A., M.D. in the years 1873-1875, with descriptions of new species. Part I. Trans. R. Ent. Soc. London 4: 267-276. 
